\title{
RELIABILITY ANALYSIS FOR MANUFACTURING GRID
}

Qiming Zou, Tao Yu, Haiyang Sun

CIMS \& Robot Center of Shanghai University, Shanghai University, China; Email: kim@staff.shu.edu.cn

Abstract: $\quad$ Manufacturing Grid (MG) emerges as a new manufacturing model to provide a general platform for networked-manufacturing. The reliability is a significant and complex issue in MG system as resources in it are geographically distributed, heterogeneous and information dynamic in nature, owned by different enterprises or organizations with their own policies. In order to make MG work efficiently, the reliability for MG had been analyzed; a hierarchical evaluation model was proposed. In the end, the use of the model was also presented in system recovery policies.

Key words: Manufacturing Grid (MG); reliability analysis; evaluation model; system recovery

\section{INTRODUCTION}

Based on the research of Networked Manufacturing, Manufacturing Grid 1 utilize not only the advanced concept and idea of Grid but also the open architecture and universal specification and tools to realize the resource sharing and cooperation for the distributed, dynamic manufacturing resources to promote the development of networked manufacturing towards openness and specification.

MG system is a distributed and heterogeneous system, in which resources are highly heterogeneous, diversity and distributed, ranging from information resources, service resources and human resources to design and manufacturing software. With MG technologies, it is possible to realize integration and optimization of all kinds of resources, provide the This work is supported by the E-institute of Shanghai Education committee-Campus Grid

Foundation of Shanghai under grant \#200302.

Please use the following format when citing this chapter:

Zou, Qiming, Yu, Tao, Sun, Haiyang, 2006, in International Federation for Information Processing (IFIP), Volume 207, Knowledge Enterprise: Intelligent Strategies In Product Design, Manufacturing, and Management, eds. K. Wang, Kovacs G., Wozny M., Fang M., (Boston: Springer), pp. 803-811. 
environment for commerce collaboration, design collaboration, manufacturing collaboration and supply chain collaboration among different enterprises, enables manufacture high quality product meet the market's requirement by lowest cost and shortest development period. However, due to the diverse failures and error conditions in the MG environments, developing, deploying and executing such activities is a challenge. Dependability is a key factor for MG. Reliability analysis and some techniques for fault tolerance are the solutions for improving the $\mathrm{MG}$ dependability.

This paper presented reliability hierarchical evaluation model for MG., a simple application case had been given out. Also, we drew the conclusion and gave out future work.

\section{RELIABILITY ANALYSIS MODEL OF MG}

\subsection{The definition of MG system reliability}

Generally speaking, the MG system reliability should include the following three aspects:

1) System Operational Reliability $\left(R_{O}\right)$ : the reliability when system is running, is a synthetic index;

2) System Inherent Reliability $\left(R_{I}\right)$ : the attributes are determined at the beginning of system is produced, there are many factors such as used material, component, design schema, hardware and software structure and manufacturing process are related to $R_{i} ;$;

3) System Uses Reliability $\left(R_{U}\right)$ : is a dynamic attribute, is related to the system's environment and human resources.

The three kinds of reliability mentioned above exists a relationship, which can be expressed with the following formula:

$$
R_{o} \approx R_{u} \square R_{I}
$$

This expression explains that Ro is decided by $R_{U}$ and $R_{F}$. To a certain system, we can acquire higher $R_{O}$ index by improving usage environment, operation and maintenance level. For the MG system, the most important reliability index is $R_{O}$. So we define MG system reliability as follows:

The ability of completing specified functions consistently under various conditional factors during the whole running process. 


\subsection{Reliability Analysis Model of MG}

\subsubsection{The running model of MG}

Now we present our running model of MG. As depicted in Figure.1, MG system is constructed on the basis of grid platform Globus. User can access the sharing resources and services through MG portal, which is the bridge between user and MG system. The portal provides many functions including information service, interface service and user authentication. The various middleware of MG system is running on the database server and application server, its function mainly provide support of system and data. The sharing resources in manufacturing industry are encapsulated as service node according to the rule and standard of grid service; the resource management is responsible for autonomy management; the resource monitor transfer dynamic status to MG system.

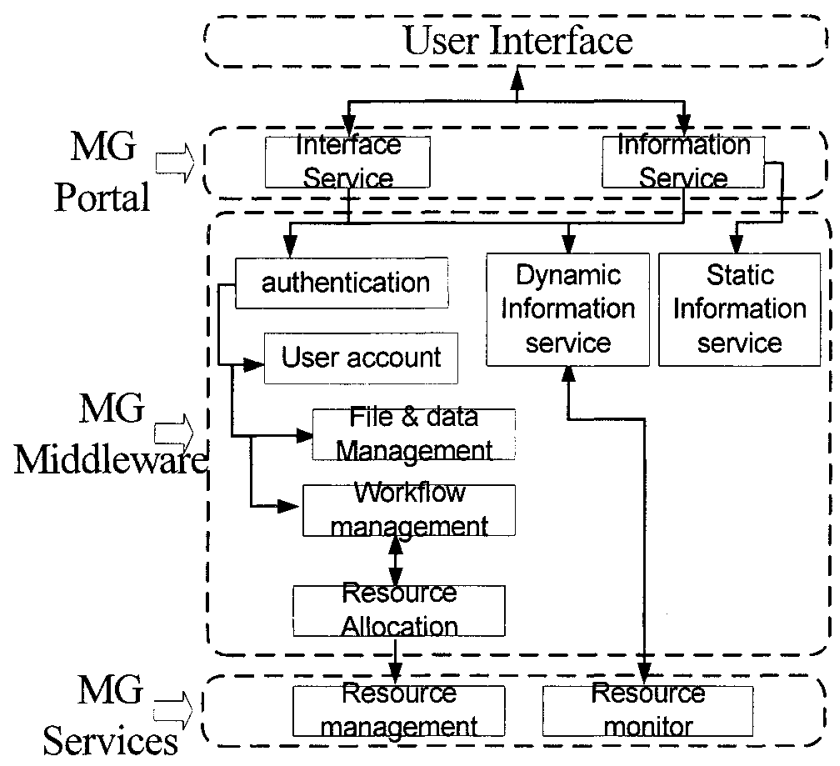

Figure 1. The running model of MG 


\subsubsection{The reliability block diagram (RBD) of MG}

The reliability block diagram analysis is a kind of analysis method of studying the logic relation of system and parts. The RBD of MG system has taken the consideration of failure or expiration of each job element.

From the running model of MG, we knew that MG portal is the entrance which user access the MG, is also the bottleneck of MG, so it' $s$ important to guarantee the MG operating persistently and steadily. Therefore, at the time of carrying on monitoring and management, we adopt hardware redundancy method to raise its reliability. The relation of two portals is parallel. Every kind of application server and database server in system enable system properly running. If there is a server breakdown, then the whole system will breakdown, so the relationship of two kinds of server is series. The service nodes in MG are distributed and independent, so their relation is parallel. The RBD of MG is illustrated in Figure. 2.

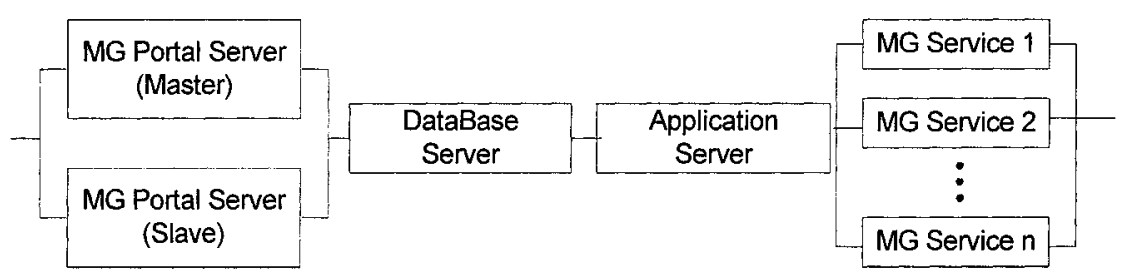

Figure 2. The RBD of MG

The reliability of MG can be expressed with the following formula:

$$
R_{M G}=R_{S Y S} \square R_{\text {Service }}=\left(R_{\text {Poralal }} \square R_{S y \text { s }}\right) \square R_{\text {Service }}
$$

Where $R_{s y s}$ denotes system resource reliability and $R_{\text {service }}$ denotes reliability of each element in MG.

\subsubsection{Hierarchical Reliability Evaluation Model of MG}

As mentioned above, MG is a complex system, from the view of reliability analysis, can be divided into four levels, as depicted in Figure.3. The four levers are system level, sub-system level, child-system level and element level. 


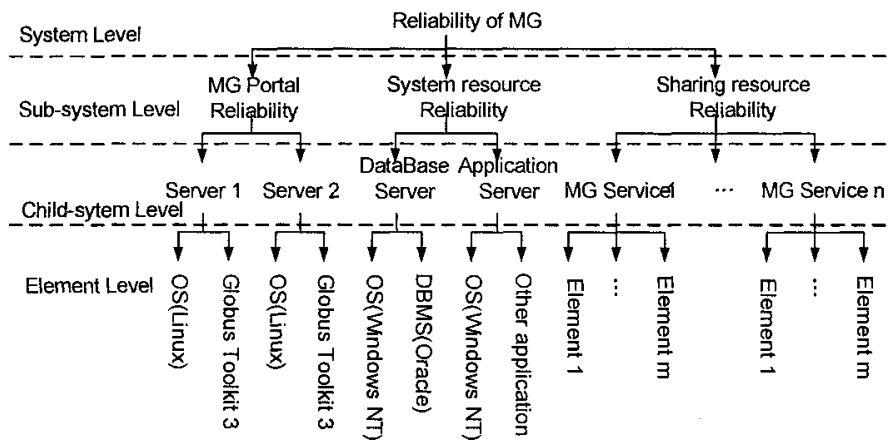

Figure 3. Hierarchical reliability evaluation model of $\mathrm{MG}$

The child system, which is one element in MG, is independent to some degree, also is a integration as a whole for MG. First of all, the reliability for child system is very important to reliability analysis for MG. An element's reliability evaluation model (REM) can be expressed with

$$
R E M=\left(V, V S, \Gamma_{v}\right)
$$

Where $V=\left\{v_{1}, v_{2}, \cdots, v_{n}\right\}$ is a group of variables that stand for object running status; $V S=\{0,1,2,3\}$ is variable status space; while $\Gamma v$ is a reflection relation from $\mathrm{V}$ to $\mathrm{VS}$.

The MG reliability evaluation model (MGREM) is based on element's REM, so it can be formally expressed with $M G R E M=f\left(\mathrm{rem}_{1}, r \mathrm{em}_{2}, \cdots, r e \mathrm{~m}_{\mathrm{n}}\right)$, where rem $_{i}$ denotes $i$-th element's reliability. The influence degree of various element is different, so must consider the weight index $\lambda_{i} \quad(i=1,2, \cdots, n)$, and $\sum_{i=1}^{n} \lambda_{i}=1, \lambda=\left(\lambda_{1}, \lambda_{2}, \cdots \lambda_{m}\right)$ is the volume of reliability evaluation. So the MGREM can be computed as:

$$
M G R E M=f\left(\mathrm{nem}_{1}, \mathrm{rem}_{2}, \cdots, r \mathrm{em}_{h}\right)=\sum_{i=1}^{n} \lambda_{i} \mathrm{rem} / n
$$

In the reliability analysis for single element process, status parameters are real-time variables from running element, while performance parameters can trace running status of element. There is a mapping relation to be decided between above two parameters. For different element, their parameters values are different and special. When the performance 
parameters of element are used to reliability analysis, the result can be used in the super level reliability analysis.

In the following example, we adapt reliability level and average life to illustrate the MGREM. Suppose that $k(k=1,2, \cdots, n)$ is element number, $i \in V S(i=0,1,2,3)$ is the status number; $v_{k}\left(v_{k} \in\{0,1,2,3\}\right)$ is the status variable of element in MG; $y(y \in\{0,1,2,3\})$ is the status variable of MG system.

From Figure.3, we knew that MG system is made up of three subsystems. Its status is decided by the worst system's status $y=\min \left\{v_{1}, v_{2}, v_{3}\right\}$.

Suppose that $p(t, i)$ and $p_{k}(t, i)$ denotes the probability located in state $i$ and time $t$ of MG respectively, where $i=0,1,2,3,4, t \in[0, \infty]$; the whole MG system and $k$-th element performance reliability are $R(t, i)$ and $R_{k}(t, i)$, can be computed as:

$$
\begin{aligned}
& R(t, i)=p\{y(t) \geq i, t \in[0, \infty)\}=\sum_{j=i}^{m} p(t, j) \\
& R_{k}(t, i)=\sum_{j=1}^{m} p_{k}(t, j)
\end{aligned}
$$

where $\sum_{i=0}^{m} p(t, i)=1, \sum_{i=0}^{m} p_{k}(t, i)=1, \quad 1=R(t, 0) \geq R(t, 1) \geq R(t, 2) \geq \cdots \geq R(t, m)$. When $i=m$, then the equation $p(t, m)=R(t, m)$ is correct. According to the definition of $p(t, i)$ and $R(t, i)$, the MG system average occupied time $M_{s}(i)$ and average life $\theta(i) \quad(i=[i, m])$ at the state $i$ can be computed respectively as follows:

$$
M_{s}(i)=\int_{0}^{\infty} p(t, i) d t
$$




$$
\theta(i)=\int R(t, i) d t=\int_{0}^{\infty}\left[\sum_{j=i}^{m} p(t, i)\right] d t=\sum_{j=i}^{m} M_{s}(j), i=1,2 \cdots, m
$$

\section{USES OF RELIABILITY ANALYSIS MODEL}

\subsection{Reliability Analysis Process for MG}

As shown in Figure.4, there are five stages in the MG reliability analysis process: data collection, data storage \& management, data analysis and event generation, storage and handling.

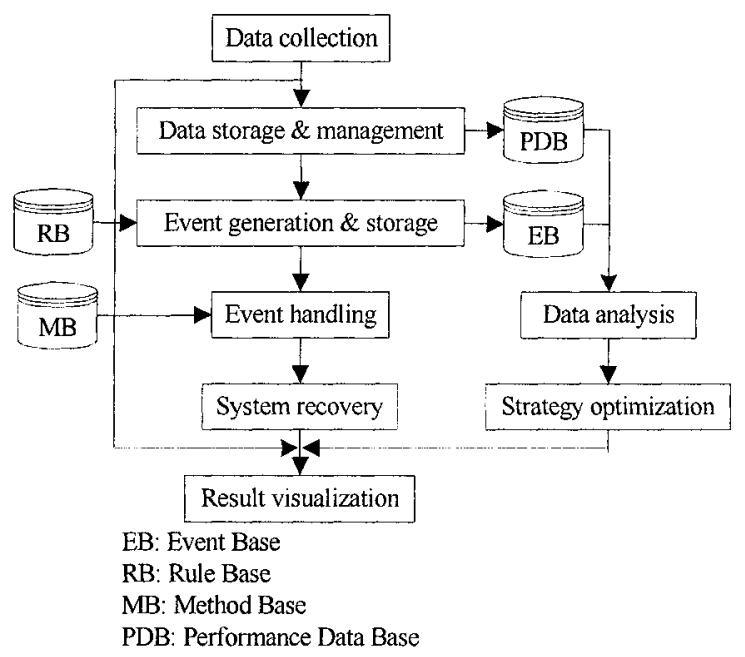

Figure 4. Reliability analysis process

The data collection module collects performance data from the managed resources, which is used for reliability evaluation. After data cleaning and storage, the event management starts to determine if there are any alert or warning events. When some events are generated, warning messages are sent to the system administrator, or corresponding recovery actions are taken if they are defined in the method base (MB). At the same time, the data analysis module extracts data from $\mathrm{PDB}$ and $\mathrm{EB}$, and performs further analysis to provide proposals for optimization of the managerial strategy or direct system recovery. The process of reliability management is a closed loop. The task of monitoring is performed from data collection to result 
visualization. Then, performance optimization is accomplished from the strategy optimization to data collection. The optimization results and performance problems are fed back after a new monitor process is started. The iterative process is a basic workflow of the reliability management.

\subsection{Recovery policy of MG}

\subsubsection{Recovery system of MG}

As shown in Figure. 5, there are key four agents in the recovery system 2,3. They are reliability manager, Mobile Agent (MA) server, MA generator and MA.

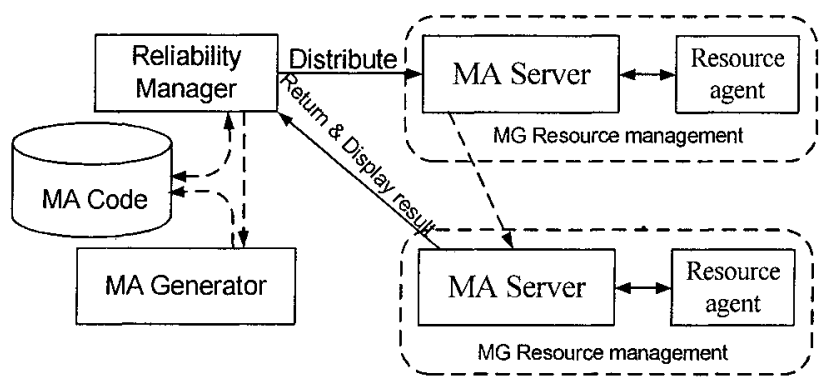

Figure 5. Recovery system of MG

G reliability manager with GUI moderates monitor and control strategy about MG resources. Manager can discover the agent process in working, maintain and update agent list.

User access MA through MA server module, which is installed on each managing resources. Logically, MA server located in the upper layer of resource agent, create effective environment for receiving, instantiation, running and scheduling MA object. It's very import that integration with resource management. There are four components in the MA server: Mobile Agent Listener, Security Facility Component, Service Facility Component and Mobile Facility Component.

Mobile Agent Generator (MAG) is an automatic tool for generating MA code in nature, can customize the combination of MA according to service requirement. The code is stored in the MA base. So MA can extend frame function dynamically, transmit initialization state of MA server.

MA is a Java object with only identifier can be transmitted among hosts, and executing particular management task in independent process. There is a routine table and a data folder in MA, the function of the folder is store and 
collects management information and method that interact with polling resources.

\subsubsection{Implementation process of recovery system}

One of the important tasks for reliability management is recover data after system had breakdown. So the prerequisite preparation should include backup data, storage changed data and trace required resources. When the events that influence integrality, consistency and validity of system data occurs, MG reliability manager get the recovery method from event handling method base according to the monitor rule, create mobile agent, then dispatch it to fault resource node, put it into event handling buffer list, wait for executing auto recovery operation or notify the related resource node to execute corresponding operation.

\section{CONCLUSIONS AND FUTURE WORK}

The generic, heterogeneous, dynamic, and high-latency nature of the MG makes it fragile, and how to measure the reliability is a key issue to provide high availability services. This paper presents a general reliability analysis model for MG, and uses of the model are also discussed. In the future, more use cases will be considered for the model. In this paper, we do not consider the dynamic attribute parameters of manufacturing resources in MG, such factors will be considered in our future model and application.

\section{REFERENCES}

1. Lilan Liu, TaoYu, Zhanbei Shi, et al, Self-organization manufacturing grid and its task scheduling algorithm, Computer Integrated Manufacturing System, 2003, 9 ( 6):449-455.

2. D.Gavalas, D.Greenwood, Advanced Network Monitoring Applications Based on Mobile/Intelligent Agent Technology, Computer Communications, 2000, (23):720-730.

3. Andre Mello Barotto, Anddriano de Souza, Carlos Becker Westphall, Distributed Network Management Using SNMP, Java, WWW and CORBA, Journal of Network and Systems Management, 2000, 8(4):483-497. 